\title{
2,3-Migration in Rh(II)-Catalyzed
}

\section{Reaction of $\beta$-Trifluoroacetamido $\alpha$-Diazocarbonyl Compounds}

\author{
Feng Xu, Shiwei Zhang, Xiangnan Wu, Yu Liu, Weifeng Shi and Jianbo Wang*
}

Beijing National Laboratory of Molecular Sciences (BNLMS), Key Laboratory of Bioorganic Chemistry and Molecular Engineering of Ministry of Education, College of Chemistry, Peking University, Beijing 100871, China

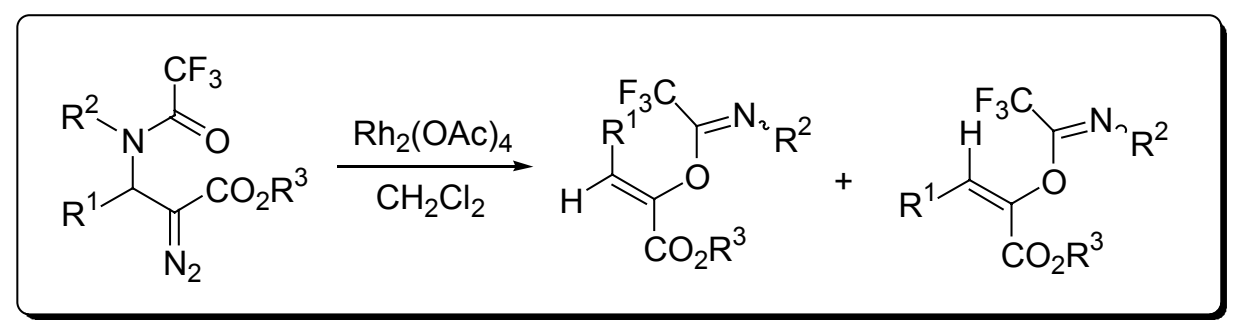

\section{Contents}

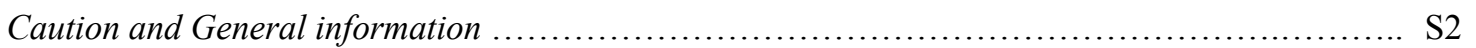

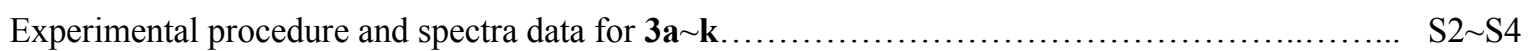

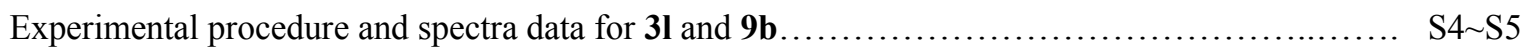

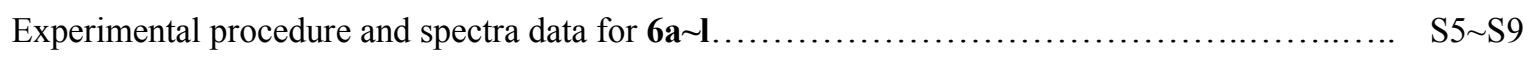

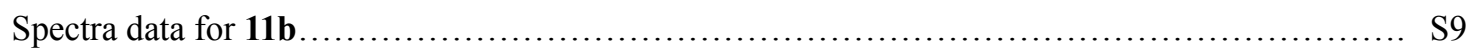

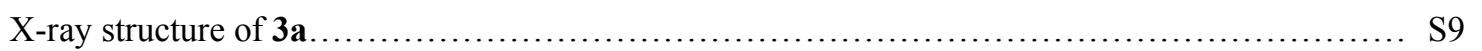

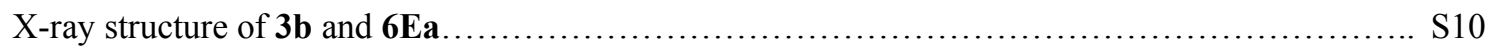

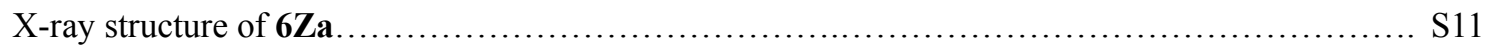


Caution: Diazo compounds are generally toxic and potentially explosive. They should be handled with care in a well-ventilated fume hood.

General information. All reactions with air- and moisture-sensitive components were performed under a nitrogen atmosphere in a flame-dried reaction flask. All solvents were distilled prior to use. $\mathrm{CH}_{2} \mathrm{Cl}_{2}$ was freshly distilled from $\mathrm{CaH}_{2}$ and THF was freshly distilled from Na before use. The boiling point of petroleum ether is between 30 and 60 ${ }^{\circ} \mathrm{C}$. For chromatography, 200-300 mesh silica gel (Qingdao, China) was employed. ${ }^{1} \mathrm{H}$ and ${ }^{13} \mathrm{C}$ NMR spectra were recorded at 200 and $50 \mathrm{MHz}$ with a Varian Mercury 200 spectrometer or 300 and $75 \mathrm{MHz}$ with a Varian Mercury 300 spectrometer. Chemical shifts are reported by ppm using tetramethylsilane as internal standard. Mass spectra were obtained on a VG ZAB-HS mass spectrometer. IR spectra were recorded with a Nicolet 5MX-S infrared spectrometer.

\section{Methyl 2-Diazo-3-[(N-phenyl)trifluoroacetamido]-3-phenylpropionate (3a)}

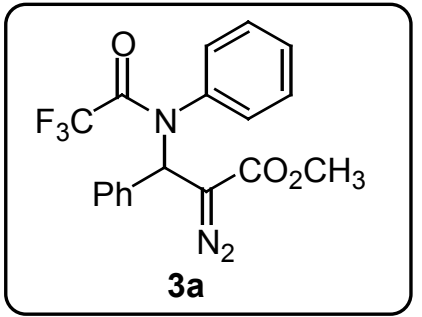

TLC $R_{f}$ (petroleum ether : acetone $\left.8: 1\right)=0.41$; IR (film) 2956, 2109, 1699, 1595, 1493, 1438, 1336, 1209, 1179, 1153, 1113, 1032, 952, 839, 742, 725, 698 $\mathrm{cm}^{-1} ;{ }^{1} \mathrm{H}$ NMR $\left(300 \mathrm{MHz}, \mathrm{CDCl}_{3}\right) \delta 3.84(\mathrm{~s}, 3 \mathrm{H}), 6.05(\mathrm{~s}, 1 \mathrm{H}), 7.14 \sim 7.42(\mathrm{~m}, 10 \mathrm{H})$; ${ }^{13} \mathrm{C}$ NMR $\left(75 \mathrm{MHz}, \mathrm{CDCl}_{3}\right) \delta 52.2,64.8,116.1\left(\mathrm{q}, J_{C-F}=287.1 \mathrm{~Hz}, \mathrm{CF}_{3}\right), 126.8$, $128.5,128.8,129.2,129.5,134.0,138.7,157.4$ (q, $\left.J_{C-C-F}=35.7 \mathrm{~Hz}\right), 166.0$; EI-MS (m/z, relative intensity): 349 [(M-28) $\left.{ }^{+}, 23\right], 290$ (13), 189 (11), 172 (100), 115 (25), 102 (15), 91 (23), 77 (74); Anal. calcd for $\mathrm{C}_{18} \mathrm{H}_{14} \mathrm{~F}_{3} \mathrm{~N}_{3} \mathrm{O}_{3}$ : C, 57.30; H, 3.74; N, 11.14. Found: C, 57.52; H, 4.04; N, 11.05 .

\section{Methyl 2-Diazo-3-[(N-phenyl)trifluoroacetamido]pentanoate (3b)}

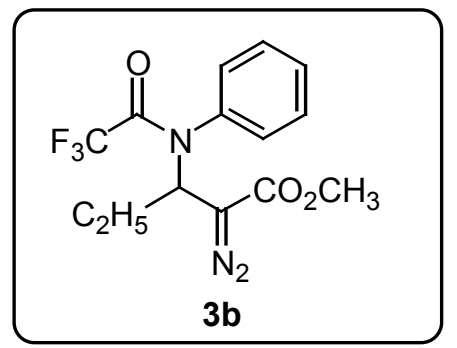

12.95 .

TLC $R_{f}$ (petroleum ether : acetone $\left.8: 1\right)=0.40$; IR (film) 2973, 2106, 1699, 1596, 1495, 1439, 1293, 1207, 1182, 1146, 1088, 962, 881, $705 \mathrm{~cm}^{-1} ;{ }^{1} \mathrm{H}$ NMR $\left(300 \mathrm{MHz} \mathrm{CDCl}_{3}\right) \delta 1.03(\mathrm{t}, J=7.2 \mathrm{~Hz}, 3 \mathrm{H}), 1.84 \sim 1.97(\mathrm{~m}, 2 \mathrm{H}), 3.82(\mathrm{~s}, 3 \mathrm{H})$, $5.13(\mathrm{~s}, 1 \mathrm{H}), 7.16 \sim 7.47(\mathrm{~m}, 5 \mathrm{H}) ;{ }^{13} \mathrm{C} \mathrm{NMR}\left(75 \mathrm{MHz}, \mathrm{CDCl}_{3}\right) \delta 10.5,22.9,52.2$, $59.2,116.1\left(\mathrm{q}, J_{C-F}=287.3 \mathrm{~Hz}, \mathrm{CF}_{3}\right), 129.3,129.4,129.6,136.8,156.6\left(\mathrm{q}, J_{C-C-F}=\right.$ $35.7 \mathrm{~Hz}), 166.2$; EI-MS ( $\mathrm{m} / \mathrm{z}$, relative intensity): 301 [(M-28) $\left.^{+}, 6\right], 272(66), 242$ (10), 189 (19), 172 (54), 144 (18), 130 (12), 113 (36), 91 (21), 77 (100); Anal. calcd for $\mathrm{C}_{14} \mathrm{H}_{14} \mathrm{~F}_{3} \mathrm{~N}_{3} \mathrm{O}_{3}$ : C, 51.07; H, 4.29; N, 12.76. Found: C, 51.08; H, 4.33; N,

Ethyl 2-Diazo-3-[(N-phenyl)trifluoroacetamido]pentanoate (3c)

TLC $R_{f}$ (petroleum ether : acetone $15: 1$ ) $=0.32$; IR (film) 2978, 2908, 1697,<smiles>CCCCCC(C(=N)OCC)N(C(=O)CC)c1ccccc1</smiles>
1596, 1495, 1373, 1207, 1182, 1146, 1085, 903, $704 \mathrm{~cm}^{-1} ;{ }^{1} \mathrm{H}$ NMR (300 MHz, $\left.\mathrm{CDCl}_{3}\right)$ $\delta 1.03(\mathrm{t}, J=7.2 \mathrm{~Hz}, 3 \mathrm{H}), 1.30(\mathrm{t}, J=7.2 \mathrm{~Hz}, 3 \mathrm{H}), 1.80 \sim 1.96(\mathrm{~m}, 2 \mathrm{H}), 4.22 \sim 4.33(\mathrm{~m}$, $2 \mathrm{H}), 5.16(\mathrm{~s}, 1 \mathrm{H}), 7.15 \sim 7.47(\mathrm{~m}, 5 \mathrm{H}) ;{ }^{13} \mathrm{C} \mathrm{NMR}\left(75 \mathrm{MHz}, \mathrm{CDCl}_{3}\right) \delta 10.5,14.4,22.9$, 59.1, 61.3, 116.1 (q, $J_{C-F}=287.3 \mathrm{~Hz}, \mathrm{CF}_{3}$ ), 129.3, 129.6, 129.6, 136.5, 136.5, 156.6 (q, $\left.J_{C-C-F}=35.6 \mathrm{~Hz}\right), 165.8$; EI-MS ( $\mathrm{m} / \mathrm{z}$, relative intensity): $315\left[(\mathrm{M}-28)^{+}, 3\right], 286(39)$, 242 (9), 189 (11), 172 (42), 144 (12), 120 (20), 99 (25), 77 (70), 69 (20), 55 (20), 29 (100); Anal. calcd for $\mathrm{C}_{15} \mathrm{H}_{16} \mathrm{~F}_{3} \mathrm{~N}_{3} \mathrm{O}_{3}$ : C, 52.48; H, 4.70; N, 12.24. Found: C, 52.50; H, 4.64; N, 12.32 . 
Ethyl 2-Diazo-3-[[N-(4-methoxyl)phenyl] trifluoroacetamido]pentanoate (3d)

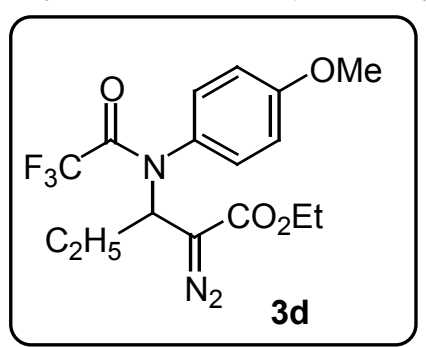

TLC $R_{f}$ (petroleum ether : acetone $\left.8: 1\right)=0.40$; IR (film) 2976, 2097, 1698, $1608,1511,1296,1253,1182,1146,1085,1033,843,739 \mathrm{~cm}^{-1} ;{ }^{1} \mathrm{H}$ NMR $(300$ $\left.\mathrm{MHz}, \mathrm{CDCl}_{3}\right) \delta 1.03(\mathrm{t}, J=7.2 \mathrm{~Hz}, 3 \mathrm{H}), 1.30(\mathrm{t}, J=7.2 \mathrm{~Hz}, 3 \mathrm{H}), 1.75 \sim 1.92(\mathrm{~m}, 2 \mathrm{H})$, $3.85(\mathrm{~s}, 3 \mathrm{H}), 4.22 \sim 4.33(\mathrm{~m}, 2 \mathrm{H}), 5.20(\mathrm{~s}, 1 \mathrm{H}), 6.92 \sim 6.95(\mathrm{~m}, 2 \mathrm{H}), 7.04 \sim 7.07(\mathrm{~m}$, $1 \mathrm{H}), 7.21 \sim 7.23(\mathrm{~m}, 1 \mathrm{H}) ;{ }^{13} \mathrm{C} \mathrm{NMR}\left(75 \mathrm{MHz}, \mathrm{CDCl}_{3}\right) \delta 10.5,14.4,23.0,55.4,58.7$, $61.2,114.3,116.2\left(\mathrm{q}, J_{C-F}=287.1 \mathrm{~Hz}, \mathrm{CF}_{3}\right), 128.6,130.6,157.0\left(\mathrm{q}, J_{C-C-F}=35.1 \mathrm{~Hz}\right)$, 160.1, 165.8; EI-MS ( $\mathrm{m} / z$, relative intensity): $345\left[(\mathrm{M}-28)^{+}, 10\right], 272(11), 219$ (100), 202 (43), 174 (8), 149 (21), 122 (25), 107 (15), 99 (53); Anal. calcd for $\mathrm{C}_{16} \mathrm{H}_{18} \mathrm{~F}_{3} \mathrm{~N}_{3} \mathrm{O}_{4}: \mathrm{C}, 51.48 ; \mathrm{H}, 4.86 ; \mathrm{N}$, 11.26. Found: C, 51.30; H, 4.89; N, 11.25.

\section{Ethyl 2-Diazo-3-[[N-(4-methoxyl)phenyl] trifluoroacetamido]nonanoate (3e)}

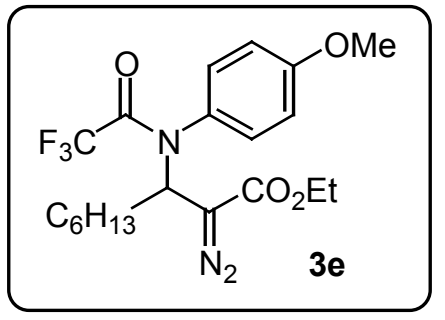

TLC $R_{f}$ (petroleum ether : acetone $8: 1$ ) $=0.34$; IR (film) 2933, 2098, 1698, $1608,1512,1298,1253,1205,1182,1153,1097, \quad 1034,841,757,739 \mathrm{~cm}^{-1} ;{ }^{1} \mathrm{H}$ NMR $\left(300 \mathrm{MHz}, \mathrm{CDCl}_{3}\right) \delta 0.89(\mathrm{t}, J=6.6 \mathrm{~Hz}, 3 \mathrm{H}), 1.23 \sim 1.43(\mathrm{~m}, 11 \mathrm{H})$, $1.75 \sim 1.82(\mathrm{~m}, 2 \mathrm{H}), 3.84(\mathrm{~s}, 3 \mathrm{H}), 4.22 \sim 4.33(\mathrm{~m}, 2 \mathrm{H}), 5.27 \sim 5.29(\mathrm{~m}, 1 \mathrm{H}), 6.92 \sim 6.95$ $(\mathrm{m}, 2 \mathrm{H}), 7.03 \sim 7.06(\mathrm{~m}, 1 \mathrm{H}), 7.19 \sim 7.22(\mathrm{~m}, 1 \mathrm{H}) ;{ }^{13} \mathrm{C}$ NMR $\left(75 \mathrm{MHz}, \mathrm{CDCl}_{3}\right)$ $\delta 13.9,14.4,22.4,25.8,28.7,29.7,31.4,55.4,56.9,61.2,114.3,116.2\left(\mathrm{q}, J_{C-F}=\right.$ $\left.286.7 \mathrm{~Hz}, \mathrm{CF}_{3}\right), 128.6,130.6,156.7$ (q, $\left.J_{C-C-F}=35.1 \mathrm{~Hz}\right), 160.1,165.8$; EI-MS $(m / z$, relative intensity): $401\left[(\mathrm{M}-28)^{+}, 11.2\right], 328$ (14), 219 (100), 202 (58), 149 (19), 109 (35); Anal. calcd for $\mathrm{C}_{20} \mathrm{H}_{26} \mathrm{~F}_{3} \mathrm{~N}_{3} \mathrm{O}_{4}$ : C, 55.94; H, 6.10; N, 9.79. Found: C, 56.16; H, 6.13; N, 9.50.

Ethyl 2-Diazo -3-[(N-4-methoxylphenyl)trifluoroacetamido]-3-Phenylpropionate(3f)<smiles>CCOC(=N)C(C(=O)OCC)N(C(=O)CC1CCCCC1)c1ccc(OC)cc1</smiles>

TLC $R_{f}$ (petroleum ether : acetone $8: 1$ ) $=0.48$; IR (film) 2982, 2109, 1698, $1607,1511,1300,1249,1207,1179,1167,1154,1109,1032,842,740,715 \mathrm{~cm}^{-1}$; ${ }^{1} \mathrm{H} \mathrm{NMR}\left(300 \mathrm{MHz}, \mathrm{CDCl}_{3}\right) \delta 1.30(\mathrm{t}, J=7.2 \mathrm{~Hz}, 3 \mathrm{H}), 3.79(\mathrm{~s}, 3 \mathrm{H}), 4.29$ (q, $J=$ $7.2 \mathrm{~Hz}, 2 \mathrm{H}), 6.09$ (s, 1H), 6.84 7.05 (m, 3H), 7.34 7.44 (m, 6H); ${ }^{13} \mathrm{C}$ NMR $(75$ $\left.\mathrm{MHz}, \mathrm{CDCl}_{3}\right) \delta 14.3,55.3,61.3,64.3,113.8,114.6,116.1\left(\mathrm{q}, J_{C-F}=287.3 \mathrm{~Hz}\right.$, $\left.\mathrm{CF}_{3}\right), 126.8,128.4,129.1,130.1,130.8,134.2,157.5\left(\mathrm{q}, J_{C-C-F}=35.7 \mathrm{~Hz}\right), 160.1$, 165.6; EI-MS ( $\mathrm{m} / z$, relative intensity): 421( $\left.\mathrm{M}^{+}, 0.6\right), 393\left[(\mathrm{M}-28)^{+}, 46\right], 347$ (16), 320 (51), 219 (51), 203 (77), 175 (59), 147 (100), 122 (23); Anal. calcd for $\mathrm{C}_{20} \mathrm{H}_{18} \mathrm{~F}_{3} \mathrm{~N}_{3} \mathrm{O}_{4}: \mathrm{C}, 57.01 ; \mathrm{H}, 4.31 ; \mathrm{N}, 9.97$. Found: C, 57.25; H, 4.35; N, 9.93.

Ethyl 2-Diazo-3-[(N-4-methoxylphenyl)trifluoroacetamido]-3-(4-phenyl)phenylpropionate (3g)<smiles>CCOC(=O)C(Cc1ccccc1)N(C(=O)C(F)(F)F)c1ccc(OC)cc1</smiles>

TLC $R_{f}$ (petroleum ether : acetone $\left.8: 1\right)=0.31$; IR (film) 2110, 1698, $1607,1511,1299,1249,1207,1180,1167,1106,1033,839,752,698 \mathrm{~cm}^{-1} ;{ }^{1} \mathrm{H}$ NMR (300 MHz, $\left.\mathrm{CDCl}_{3}\right) \delta 1.22(\mathrm{t}, J=7.2 \mathrm{~Hz}, 3 \mathrm{H}), 3.70(\mathrm{~s}, 3 \mathrm{H}), 4.19 \sim 4.26(\mathrm{~m}$, $2 \mathrm{H}), 6.03(\mathrm{~s}, 1 \mathrm{H}), 6.76 \sim 7.16(\mathrm{~m}, 3 \mathrm{H}), 7.27 \sim 7.56(\mathrm{~m}, 10 \mathrm{H}) ;{ }^{13} \mathrm{C} \mathrm{NMR}(75 \mathrm{MHz}$, $\left.\mathrm{CDCl}_{3}\right) \delta 14.4,55.4,61.4,64.2,113.9,114.7,116.2\left(\mathrm{q}, J_{C-F}=287.5 \mathrm{~Hz}, \mathrm{CF}_{3}\right)$, $127.0,127.3,127.8,128.8,130.1,130.9,133.1,140.0,141.3,157.6\left(\mathrm{q}, J_{C-C-F}=\right.$ $35.7 \mathrm{~Hz}), 160.1,165.7$; EI-MS (m/z, relative intensity): $469\left[(\mathrm{M}-28)^{+}, 100\right]$, 
423 (69), 396 (31), 354 (45), 286 (50), 202 (46); Anal. calcd for $\mathrm{C}_{26} \mathrm{H}_{22} \mathrm{~F}_{3} \mathrm{~N}_{3} \mathrm{O}_{4}$ : C, 62.77; H, 4.46; N, 8.45. Found: C, $62.58 ; \mathrm{H}, 4.74 ; \mathrm{N}, 8.19$.

Ethyl 2-Diazo-3-[(N-4-nitrophenyl)trifluoroacetamido]pentanoate (3h)

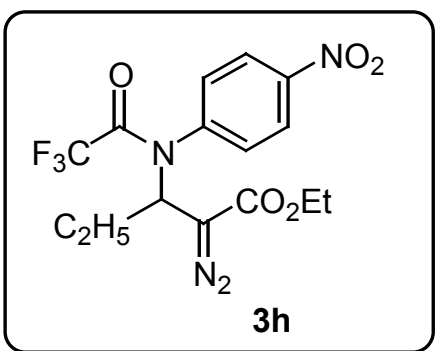

TLC $R_{f}$ (petroleum ether : acetone $\left.15: 1\right)=0.24$; IR (film) 2980, 2105, 1702, $1595,1529,1349,1291,1210,1171,1147,1086,855,704 \mathrm{~cm}^{-1} ;{ }^{1} \mathrm{H}$ NMR $(300$ $\left.\mathrm{MHz}, \mathrm{CDCl}_{3}\right) \delta 1.04(\mathrm{t}, J=7.2 \mathrm{~Hz}, 3 \mathrm{H}), 1.32(\mathrm{t}, J=7.2 \mathrm{~Hz}, 3 \mathrm{H}), 1.95 \sim 2.05(\mathrm{~m}$, $2 \mathrm{H}), 4.24 \sim 4.34(\mathrm{~m}, 2 \mathrm{H}), 4.87(\mathrm{~s}, 1 \mathrm{H}), 7.42 \sim 7.66(\mathrm{~m}, 2 \mathrm{H}), 8.32 \sim 8.35(\mathrm{~m}, 2 \mathrm{H}) ;{ }^{13} \mathrm{C}$ NMR $\left(75 \mathrm{MHz}, \mathrm{CDCl}_{3}\right) \delta 10.6,14.3,22.8,58.9,61.4,115.7\left(\mathrm{q}, J_{C-F}=287.1 \mathrm{~Hz}\right.$, $\left.\mathrm{CF}_{3}\right), 124.6,130.2,143.4,147.9,157.3\left(\mathrm{q}, J_{C-C-F}=35.7 \mathrm{~Hz}\right), 165.7$; EI-MS $(m / z$, relative intensity): 360 [(M-28) $\left.{ }^{+}, 5\right], 331$ (100), 287 (27), 217 (68), 99 (27); Anal. calcd for $\mathrm{C}_{15} \mathrm{H}_{15} \mathrm{~F}_{3} \mathrm{~N}_{4} \mathrm{O}_{5}: \mathrm{C}, 46.40 ; \mathrm{H}, 3.89 ; \mathrm{N}, 14.43$. Found: $\mathrm{C}, 46.48 ; \mathrm{H}, 3.90 ; \mathrm{N}, 14.50$.

Ethyl 2-Diazo-3-[(N-4-nitrophenyl)trifluoroacetamido]nonanoate (3i)

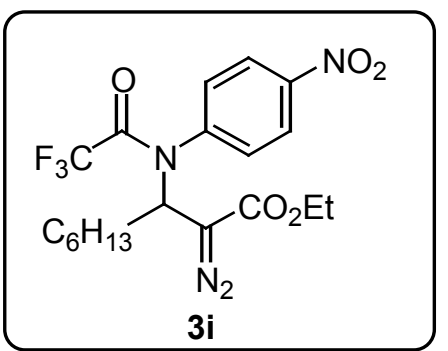

TLC $R_{f}$ (petroleum ether : acetone $\left.15: 1\right)=0.28$; IR (film) 2932, 2861, 2105, $1704,1595,1530,1348,1288,1210,1171,1153,1098,854,703 \mathrm{~cm}^{-1} ;{ }^{1} \mathrm{H}$ NMR $\left(300 \mathrm{MHz}, \mathrm{CDCl}_{3}\right) \delta 0.87(\mathrm{t}, J=6.6 \mathrm{~Hz}, 3 \mathrm{H}), 1.29 \sim 1.42(\mathrm{~m}, 11 \mathrm{H}), 1.95(\mathrm{~s}, 2 \mathrm{H})$, $4.24 \sim 4.34(\mathrm{~m}, 2 \mathrm{H}), 4.95(\mathrm{~s}, 1 \mathrm{H}), 7.42 \sim 7.65(\mathrm{~m}, 2 \mathrm{H}), 8.33 \sim 8.36(\mathrm{~m}, 2 \mathrm{H}) ;{ }^{13} \mathrm{C} \mathrm{NMR}$ $\left(75 \mathrm{MHz}, \mathrm{CDCl}_{3}\right) \delta 13.9,14.3,22.4,26.0,28.6,29.5,31.4,59.2,61.4,115.6(\mathrm{q}$, $\left.J_{C-F}=287.1 \mathrm{~Hz}, \mathrm{CF}_{3}\right), 124.7,130.2,143.5,148.0,156.3\left(\mathrm{q}, J_{C-C-F}=36.1 \mathrm{~Hz}\right)$, 165.7; EI-MS ( $m / z$, relative intensity): $416\left[(\mathrm{M}-28)^{+}, 4\right], 387(10), 343$ (39), 331 (66), 299 (20), 234 (23), 217 (100), 109 (52); Anal. calcd for $\mathrm{C}_{19} \mathrm{H}_{23} \mathrm{~F}_{3} \mathrm{~N}_{4} \mathrm{O}_{5}$ : C, 51.35; H, 5.22; N, 12.61. Found: C, $51.60 ; \mathrm{H}, 5.33 ; \mathrm{N}, 12.31$.

Ethyl 2-Diazo-3-[(N-4-nitrophenyl)trifluoroacetamido]-3-phenylpropionate (3j)

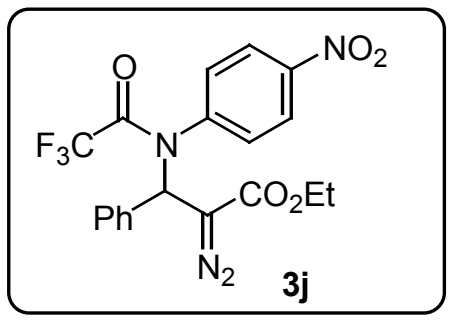

TLC $R_{f}$ (petroleum ether : acetone $8: 1$ ) $=0.45$; IR (film) 2986, 2111, 1705, $1595,1529,1348,1213,1168,1108,1017,855,729,699 \mathrm{~cm}^{-1} ;{ }^{1} \mathrm{H}$ NMR $(300$ $\left.\mathrm{MHz}, \mathrm{CDCl}_{3}\right) \delta 1.33(\mathrm{t}, J=7.2 \mathrm{~Hz}, 3 \mathrm{H}), 4.29 \sim 4.38(\mathrm{~m}, 2 \mathrm{H}), 5.90(\mathrm{~s}, 1 \mathrm{H})$, 7.33 7.48 (m, 7H), 8.27 8.29 (m, 2H) ${ }^{13} \mathrm{C}$ NMR (75 MHz, $\left.\mathrm{CDCl}_{3}\right) \delta 14.4,61.5$, $66.0,115.8\left(\mathrm{q}, J_{C-F}=287.3 \mathrm{~Hz}, \mathrm{CF}_{3}\right), 124.7,126.7,130.0,129.5,133.2,144.6$, 148.0, $156.9\left(\mathrm{q}, J_{C-C-F}=36.7 \mathrm{~Hz}\right), 165.6$; EI-MS ( $\mathrm{m} / \mathrm{z}$, relative intensity): 408 $\left[(\mathrm{M}-28)^{+}, 41\right], 335$ (19), 234 (14), 217 (100), 147 (28), 122 (15), 103 (41), 91 (25), 77 (28); Anal. calcd for $\mathrm{C}_{19} \mathrm{H}_{15} \mathrm{~F}_{3} \mathrm{~N}_{4} \mathrm{O}_{5}$ : C, 52.30; H, 3.46; N, 12.84. Found: C, 52.38; H, 3.59; N, 12.78.

\section{Ethyl 2-Diazo-3-[(N-3,4-dichlorophenyl)trifluoroacetamido]pentanoate (3k)}

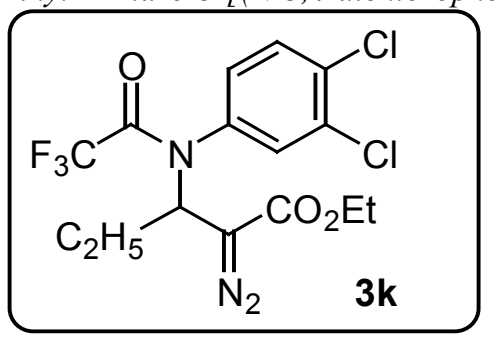

TLC $R_{f}$ (petroleum ether : acetone $\left.30: 1\right)=0.21$; IR (film) 2979, 2107, $1702,1588,1561,1473,1373,1292,1211,1178,1149,1086,910,806,738$ $\mathrm{cm}^{-1} ;{ }^{1} \mathrm{H}$ NMR $\left(300 \mathrm{MHz}, \mathrm{CDCl}_{3}\right) \delta 1.03(\mathrm{t}, J=7.2 \mathrm{~Hz}, 3 \mathrm{H}), 1.31(\mathrm{t}, J=7.2$ $\mathrm{Hz}, 3 \mathrm{H}), 1.94$ (s, 2H), 4.28 (q, $J=7.2 \mathrm{~Hz}, 2 \mathrm{H}), 4.91(\mathrm{~s}, 1 \mathrm{H}), 7.05 \sim 7.56(\mathrm{~m}, 3 \mathrm{H})$; ${ }^{13} \mathrm{C}$ NMR $\left(75 \mathrm{MHz}, \mathrm{CDCl}_{3}\right) \delta 10.6,14.4,22.8,58.8,61.4,115.8$ (q, $J_{C-F}=$ 
$\left.287.1 \mathrm{~Hz}, \mathrm{CF}_{3}\right), 128.6,130.8,131.2,134.2,136.7,156.9$ (q, $\left.J_{C-C-F}=35.7 \mathrm{~Hz}\right), 165.67$; EI-MS ( $\mathrm{m} / z$, relative intensity): 383 [(M-28) , 3], 354 (23), 257 (9), 240 (27), 145 (18), 127 (15), 99 (61); Anal. calcd for $\mathrm{C}_{15} \mathrm{H}_{14} \mathrm{Cl}_{2} \mathrm{~F}_{3} \mathrm{~N}_{3} \mathrm{O}_{3}: \mathrm{C}, 43.71$; H, 3.42; N, 10.19. Found: C, 43.65; H, 3.40; N, 9.98.

Ethyl 2-Diazo-3-[(N-3,4-dichlorophenyl)trifluoroacetamido]-3-phenylpropionate (3l)

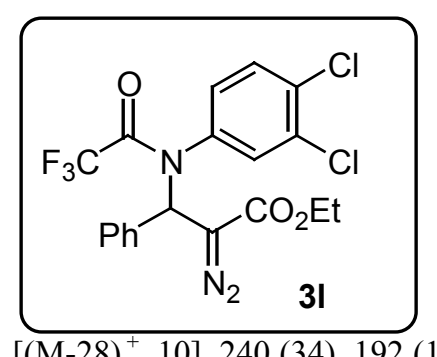

$\left[(\mathrm{M}-28)^{+}, 10\right], 240$ (34), 192 (10), 175 (12), 147 (34), 105 (100), 77 (30; Anal. calcd for $\mathrm{C}_{19} \mathrm{H}_{14} \mathrm{Cl}_{2} \mathrm{~F}_{3} \mathrm{~N}_{3} \mathrm{O}_{3}$ : C, 49.58; H, 3.07; N, 9.13. Found: C, 49.40; H, 3.25; N, 8.99.

TLC $R_{f}$ (petroleum ether : acetone $15: 1$ ) = 0.43; IR (film) 2982, 2113, 1705, $1586,1563,1472,1373,1319,1213,1172,1155,1107,1016,823,740,723,705$ $\mathrm{cm}^{-1} ;{ }^{1} \mathrm{H}$ NMR $\left(300 \mathrm{MHz}, \mathrm{CDCl}_{3}\right) \delta 1.32(\mathrm{t}, J=7.2 \mathrm{~Hz}, 3 \mathrm{H}), 4.28 \sim 4.36(\mathrm{~m}, 2 \mathrm{H})$, $5.89(\mathrm{~s}, 1 \mathrm{H}), 7.28 \sim 7.49(\mathrm{~m}, 8 \mathrm{H}) ;{ }^{13} \mathrm{C}$ NMR $\left(75 \mathrm{MHz}, \mathrm{CDCl}_{3}\right) \delta 14.4,61.5,65.7$, 115.9 (q, $J_{C-F}=287.3 \mathrm{~Hz}, \mathrm{CF}_{3}$ ), 126.7, 128.3, 128.5, 129.4, 130.8, 133.4, 134.2, 138.2, $157.1\left(\mathrm{q}, J_{C-C-F}=36.1 \mathrm{~Hz}\right), 165.54$; EI-MS $(\mathrm{m} / z$, relative intensity): 431

Ethyl 2-Diazo-3-[(N-methyl)trichloroacetamido]pentanoate (9b)

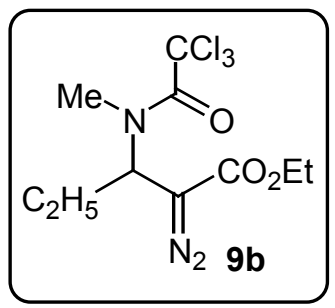

TLC $R_{f}$ (petroleum ether : acetone $15: 1$ ) = 0.44; IR (film) 2976, 2101, 1689, 1463, $1371,1319,1243,1148,1075,951,913,843,811,746 \mathrm{~cm}^{-1} ;{ }^{1} \mathrm{H}$ NMR $\left(300 \mathrm{MHz}, \mathrm{CDCl}_{3}\right)$ $\delta 1.02(\mathrm{t}, J=7.2 \mathrm{~Hz}, 3 \mathrm{H}), 1.29(\mathrm{t}, J=7.2 \mathrm{~Hz}, 3 \mathrm{H}), 1.81 \sim 2.08(\mathrm{~m}, 2 \mathrm{H}), 3.05(\mathrm{~s}, 3 \mathrm{H})$, 4.18 4.30 (m, 2H), $4.53(\mathrm{~s}, 1 \mathrm{H}) ;{ }^{13} \mathrm{C}$ NMR $\left(75 \mathrm{MHz}, \mathrm{CDCl}_{3}\right) \delta$ 10.6, 14.3, 21.9, 38.5, 61.0, 93.4, 160.2, 166.3; EI-MS ( $\mathrm{m} / z$, relative intensity): 301 [(M-28) $\left.{ }^{+}, 1\right], 272$ (82), 244 (18), 237 (16), 209 (13), 158 (18), 117 (20), 99 (21), 82 (15), 55 (18), 29 (100); Anal. calcd for $\mathrm{C}_{10} \mathrm{H}_{14} \mathrm{Cl}_{3} \mathrm{~N}_{3} \mathrm{O}_{3}: \mathrm{C}, 36.33 ; \mathrm{H}, 4.27 ; \mathrm{N}, 12.71$. Found: $\mathrm{C}, 36.30 ; \mathrm{H}, 4.28 ; \mathrm{N}, 12.68$.

(Z)-Methyl 2-(2,2,2-Trifluoro-N-phenylacetimidoyloxy)3-phenyl-2-propenoate (6Za).

TLC $R_{f}$ (petroleum ether : acetone $30: 1$ ) = 0.39; IR (film) 2954, 1729, 1651, 1597,

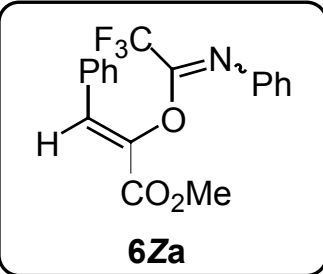

C, $62.03 ; \mathrm{H}, 4.13 ; \mathrm{N}, 3.87$. $1489,1450,1437,1321,1269,1209,1162,1128,1114,1072,971,920,756,690 \mathrm{~cm}^{-1} ;{ }^{1} \mathrm{H}$ NMR $\left(300 \mathrm{MHz}, \mathrm{CDCl}_{3}\right) \delta 3.83(\mathrm{~s}, 3 \mathrm{H}), 6.70 \sim 6.72(\mathrm{~m}, 2 \mathrm{H}), 7.04 \sim 7.18(\mathrm{~m}, 4 \mathrm{H})$, $7.41 \sim 7.63(\mathrm{~m}, 5 \mathrm{H}) ;{ }^{13} \mathrm{C}$ NMR $\left(75 \mathrm{MHz}, \mathrm{CDCl}_{3}\right) \delta 52.7,119.6,124.7,126.2,128.5,128.9$, 130.16, 131.6, 137.5, 142.6, 162.1; EI-MS ( $m / z$, relative intensity): $349\left(\mathrm{M}^{+}, 25\right), 172$ (100), 86 (11), 77 (89); Anal. calcd for $\mathrm{C}_{18} \mathrm{H}_{14} \mathrm{~F}_{3} \mathrm{NO}_{3}: \mathrm{C}, 61.89 ; \mathrm{H}, 4.04 ; \mathrm{N}, 4.01$. Found:

(E)-Methyl 2-(2,2,2-Trifluoro-N-phenylacetimidoyloxy)3-phenyl-2-propenoate (6Ea).

TLC $R_{f}$ (petroleum ether : acetone $30: 1$ ) = 0.46; IR (film) 2954, 1727, 1597, 1488,

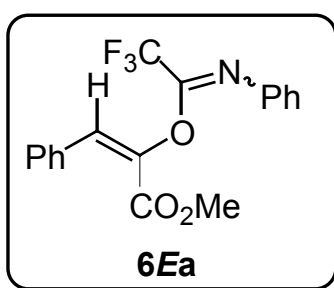

C, 62.05; H, 4.09; N, 3.92 . 1437, 1328, 1208, 1162, 1130, 1130, 931, 777, 754, 717, $694 \mathrm{~cm}^{-1} ;{ }^{1} \mathrm{H}$ NMR $(300 \mathrm{MHz}$, $\left.\mathrm{CDCl}_{3}\right) \delta 3.71(\mathrm{~s}, 3 \mathrm{H}), 6.81 \sim 6.84(\mathrm{~m}, 2 \mathrm{H}), 7.00(\mathrm{~s}, 1 \mathrm{H}), 7.09 \sim 7.14(\mathrm{~m}, 1 \mathrm{H}), 7.25 \sim 7.34(\mathrm{~m}$, $7 \mathrm{H}) ;{ }^{13} \mathrm{C}$ NMR (75 MHz, $\mathrm{CDCl}_{3}$ ) $\delta 52.1,114.1,119.6,124.7,128.0,128.7,129.0,129.5$, 131.6, 138.3, 143.0, 161.5; EI-MS ( $\mathrm{m} / \mathrm{z}$, relative intensity): 349 (M+16), 172 (95), 118 (10), 86 (21), 77 (100); Anal. calcd for $\mathrm{C}_{18} \mathrm{H}_{14} \mathrm{~F}_{3} \mathrm{NO}_{3}$ : C, 61.89; H, 4.04; N, 4.01. Found: 
(Z)-Methyl 2-(2,2,2-Trifluoro- $N$-phenylacetimidoyloxy)-2-pentenoate (6Zb).

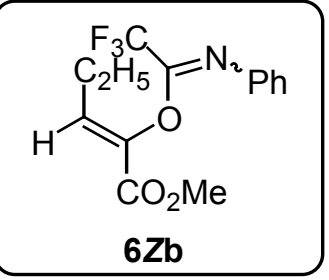

TLC $R_{f}$ (petroleum ether : acetone $\left.15: 1\right)=0.46$; IR (film) 2974, 1729, 1657, 1598, 1489, 1438, 1361, 1332, 1229, 1208, 1133, 1074, 1029, 932, 777, 754, $694 \mathrm{~cm}^{-1} ;{ }^{1} \mathrm{H}$ NMR $\left(300 \mathrm{MHz}, \mathrm{CDCl}_{3}\right) \delta 1.04(\mathrm{~s}, 3 \mathrm{H}), 2.54(\mathrm{~s}, 2 \mathrm{H}), 3.80(\mathrm{~s}, 3 \mathrm{H}), 6.05(\mathrm{~s}, 1 \mathrm{H}), 6.77 \sim 6.79(\mathrm{~m}$, 2H), 7.06 7.11 (m, 1H), 7.24 7.30 (m, 2H); ${ }^{13} \mathrm{C}$ NMR $\left(75 \mathrm{MHz}, \mathrm{CDCl}_{3}\right) \delta$ 13.4, 20.3, 52.0, 119.6, 124.4, 128.6, 135.8, 137.8, 143.2, 161.5; EI-MS ( $\mathrm{m} / z$, relative intensity): $301\left(\mathrm{M}^{+}, 5\right)$, 272 (26), 172 (100), 119 (15), 88 (58), 77 (90); Anal. calcd for $\mathrm{C}_{14} \mathrm{H}_{14} \mathrm{~F}_{3} \mathrm{NO}_{3}: \mathrm{C}, 55.82 ; \mathrm{H}$, $4.68 ; \mathrm{N}, 4.65$. Found: C, 55.87; H, 4.62; N, 4.75.

(E)-Methyl 2-(2,2,2-Trifluoro-N-phenylacetimidoyloxy)-2-pentenoate (6Eb).

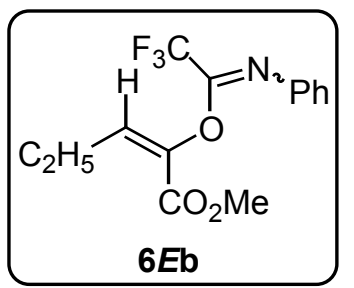

4.65. Found: $\mathrm{C}, 55.92 ; \mathrm{H}, 4.65 ; \mathrm{N}, 4.56$.

TLC $R_{f}$ (petroleum ether : acetone $15: 1$ ) = 0.41; IR (film) 2974, 1736, 1668, 1598, 1489, 1439, 1328, 1304, 1249, 1209, 1132, 1114, 1074, 971, 754, $694 \mathrm{~cm}^{-1}$; ${ }^{1} \mathrm{H}$ NMR $\left(300 \mathrm{MHz}, \mathrm{CDCl}_{3}\right) \delta 1.07(\mathrm{t}, J=7.2 \mathrm{~Hz}, 3 \mathrm{H}), 2.20 \sim 2.30(\mathrm{~m}, 2 \mathrm{H}), 3.77(\mathrm{~s}, 3 \mathrm{H}), 6.51(\mathrm{~s}$, $1 \mathrm{H}), 6.78 \sim 6.81(\mathrm{~m}, 2 \mathrm{H}), 7.07 \sim 7.29(\mathrm{~m}, 3 \mathrm{H}) ;{ }^{13} \mathrm{C} \mathrm{NMR}\left(75 \mathrm{MHz}, \mathrm{CDCl}_{3}\right) \delta 12.5,19.3$, $52.3,119.5,124.5,128.5,133.0,138.4,143.1,161.5$; EI-MS ( $\mathrm{m} / z$, relative intensity): 301 $\left(\mathrm{M}^{+}, 8\right), 272$ (12), 172 (100), 77 (69); Anal. calcd for $\mathrm{C}_{14} \mathrm{H}_{14} \mathrm{~F}_{3} \mathrm{NO}_{3}: \mathrm{C}, 55.82 ; \mathrm{H}, 4.68 ; \mathrm{N}$,

(Z)-Ethyl 2-(2,2,2-Trifluoro-N-phenylacetimidoyloxy)-2-pentenoate (6Zc).

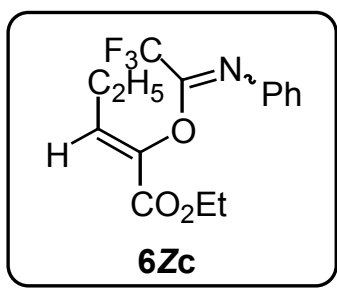

TLC $R_{f}$ (petroleum ether : acetone $15: 1$ ) = 0.54; IR (film) 2982, 1724, 1598, 1489, 1374, 1332, 1227, 1207, 1134, 1074, 1033, 923, 777, $694 \mathrm{~cm}^{-1} ;{ }^{1} \mathrm{H}$ NMR $(300 \mathrm{MHz}$, $\left.\mathrm{CDCl}_{3}\right) \delta 1.04(\mathrm{~s}, 3 \mathrm{H}), 1.32(\mathrm{t}, J=7.2 \mathrm{~Hz}, 3 \mathrm{H}), 2.55(\mathrm{~s}, 2 \mathrm{H}), 4.25$ (q, $\left.J=7.2 \mathrm{~Hz}, 2 \mathrm{H}\right), 6.04$ (s, 1H), 6.78 6.81 (m, 2H), 7.07 7.12 (m, 1H), 7.25 7.30 (m, 1H); ${ }^{13} \mathrm{C}$ NMR $(75 \mathrm{MHz}$, $\left.\mathrm{CDCl}_{3}\right) \delta 13.5,14.0,20.3,61.3,119.7,124.4,128.6,135.7,138.0,143.3,161.0$; EI-MS $\left(\mathrm{m} / \mathrm{z}\right.$, relative intensity): $315\left(\mathrm{M}^{+}, 6\right), 286(48), 172$ (100), 77 (96); Anal. calcd for $\mathrm{C}_{15} \mathrm{H}_{16} \mathrm{~F}_{3} \mathrm{NO}_{3}: \mathrm{C}, 57.14 ; \mathrm{H}, 5.12 ; \mathrm{N}, 4.44$. Found: C, 57.13; H, 5.12; N, 4.42.<smiles>CCOC(OCC)=C(CC)OC(C)=Nc1ccccc1</smiles>

TLC $R_{f}$ (petroleum ether : acetone $15: 1$ ) = 0.51; IR (film) 2978, 1730, 1669, 1598, 1489, 1328, 1300, 1277, 1248, 1208, 1161, 1133, 1114, 1073, 1026, 922, 780, 753, 694 $\mathrm{cm}^{-1} ;{ }^{1} \mathrm{H}$ NMR $\left(300 \mathrm{MHz}, \mathrm{CDCl}_{3}\right) \delta 1.07(\mathrm{t}, J=7.2 \mathrm{~Hz}, 3 \mathrm{H}), 1.29(\mathrm{t}, J=7.2 \mathrm{~Hz}, 3 \mathrm{H})$, $2.23 \sim 2.27$ (m, 2H), 4.23 (q, $J=7.2 \mathrm{~Hz}, 2 \mathrm{H}), 6.52(\mathrm{~s}, 1 \mathrm{H}), 6.79 \sim 6.82(\mathrm{~m}, 2 \mathrm{H}), 7.07 \sim 7.29$ $(\mathrm{m}, 3 \mathrm{H}) ;{ }^{13} \mathrm{C}$ NMR $\left(75 \mathrm{MHz}, \mathrm{CDCl}_{3}\right) \delta 12.5,14.0,19.3,61.5,119.5,124.5,128.5,132.6$, 138.7, 143.1, 161.0; EI-MS ( $\mathrm{m} / \mathrm{z}$, relative intensity): $315\left(\mathrm{M}^{+}, 7\right), 286$ (13), 172 (100), 77 (60); Anal. calcd for $\mathrm{C}_{15} \mathrm{H}_{16} \mathrm{~F}_{3} \mathrm{NO}_{3}: \mathrm{C}, 57.14 ; \mathrm{H}, 5.12 ; \mathrm{N}, 4.44$. Found: $\mathrm{C}, 57.14 ; \mathrm{H}, 5.14 ; \mathrm{N}, 4.41$. 
(Z)-Ethyl 2-[2,2,2-Trifluoro-N-(4-methoxyl)phenylacetimidoyloxy]-2-pentenoate (6Zd).

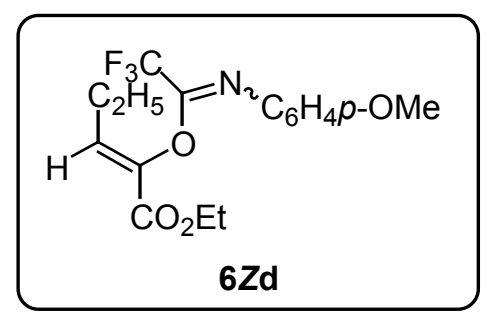

TLC $R_{f}$ (petroleum ether : acetone $8: 1$ ) =0.66; IR (film) 2981, 1726, 1657, $1608,1506,1331,1292,1228,1206,1135,1034,924,835,755 \mathrm{~cm}^{-1} ;{ }^{1} \mathrm{H}$ NMR $\left(300 \mathrm{MHz}, \mathrm{CDCl}_{3}\right) \delta 1.03 \sim 1.12(\mathrm{~m}, 3 \mathrm{H}), 1.31(\mathrm{t}, J=7.2 \mathrm{~Hz}, 3 \mathrm{H}), 2.54(\mathrm{~s}, 2 \mathrm{H})$, $3.78(\mathrm{~s}, 3 \mathrm{H}), 4.25(\mathrm{q}, J=7.2 \mathrm{~Hz}, 2 \mathrm{H}), 6.01(\mathrm{t}, J=7.5 \mathrm{~Hz}, 1 \mathrm{H}), 6.82(\mathrm{~s}, 4 \mathrm{H}) ;{ }^{13} \mathrm{C}$ NMR $\left(75 \mathrm{MHz}, \mathrm{CDCl}_{3}\right) \delta 13.5,14.0,20.3,55.3,61.3,113.9,118.2,121.8$, 135.0, 138.2, 157.0, 161.1; EI-MS ( $\mathrm{m} / \mathrm{z}$, relative intensity): $345\left(\mathrm{M}^{+}, 5\right), 219$ (100), 202 (37), 122 (67), 109 (177), 71 (76); Anal. calcd for $\mathrm{C}_{16} \mathrm{H}_{18} \mathrm{~F}_{3} \mathrm{NO}_{4}$ : C, 55.65; H, 5.25; N, 4.06. Found: C, $55.62 ; \mathrm{H}, 5.19 ; \mathrm{N}, 4.09$.

(Z)-Ethyl 2-[2,2,2-Trifluoro-N-(4-methoxylphenyl)acetimidoyloxy]-2-nonenoate (6Ze).

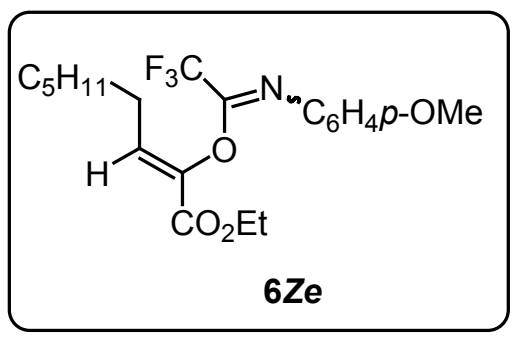

TLC $R_{f}$ (petroleum ether : acetone $\left.60: 1\right)=0.39$; IR (film) 2931, 2859, $1727,1655,1608,1506,1446,1330,1291,1241,1206,1136,1035,927$, 835, $755 \mathrm{~cm}^{-1} ;{ }^{1} \mathrm{H}$ NMR $\left(300 \mathrm{MHz}, \mathrm{CDCl}_{3}\right) \delta 0.88(\mathrm{t}, J=6.6 \mathrm{~Hz}, 3 \mathrm{H})$, $1.28 \sim 1.40(\mathrm{~m}, 11 \mathrm{H}), 2.53(\mathrm{~s}, 2 \mathrm{H}), 3.78(\mathrm{~s}, 3 \mathrm{H}), 4.24(\mathrm{q}, J=7.2 \mathrm{~Hz}, 2 \mathrm{H})$, $6.03(\mathrm{t}, J=7.8 \mathrm{~Hz}, 1 \mathrm{H}), 6.82(\mathrm{~s}, 4 \mathrm{H}) ;{ }^{13} \mathrm{C} \mathrm{NMR}\left(75 \mathrm{MHz}, \mathrm{CDCl}_{3}\right) \delta 14.0$, 25.5, 26.7, 28.9, 29.0, 31.5, 55.3, 61.2, 113.9, 121.5, 133.8, 136.1, 138.5, 156.9, 161.1; EI-MS ( $\mathrm{m} / \mathrm{z}$, relative intensity): $401\left(\mathrm{M}^{+}, 11\right), 219(15), 202$ (100), 122 (8), 107 (17); Anal. calcd for $\mathrm{C}_{20} \mathrm{H}_{26} \mathrm{~F}_{3} \mathrm{NO}_{4}$ : C, 59.84; H, 6.53; N, 3.49. Found: C, 59.95; H, 6.55; N, 3.39.

(Z/E)-Methyl 2-[2,2,2-trifluoro-N-(4-methoxylphenyl)acetimidoyloxy]-3-phenyl-2-propenoate (6Zf/6Ef).

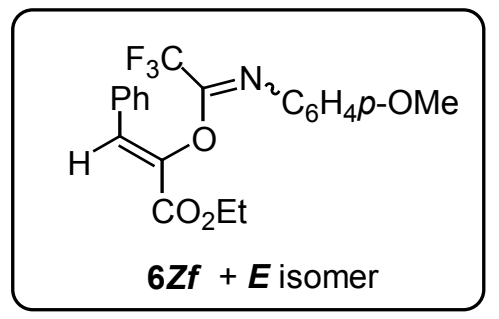

An inseparable mixture; TLC $R_{f}$ (petroleum ether : acetone $\left.10: 1\right)=0.44$; ${ }^{1} \mathrm{H}$ NMR $\left(200 \mathrm{MHz}, \mathrm{CDCl}_{3}\right) \delta 1.23(\mathrm{t}, J=7.2 \mathrm{~Hz}, 1.8 \mathrm{H}, E), 1.31(\mathrm{t}, J=7.2 \mathrm{~Hz}$, $3 \mathrm{H}, Z), 3.73 \sim 3.75(\mathrm{~m}, 4.7 \mathrm{H}, E$ and $Z), 4.19$ (q, $J=7.2 \mathrm{~Hz}, 1.1 \mathrm{H}, E), 4.25$ (q, $J=$ $7.2 \mathrm{~Hz}, 2 \mathrm{H}, Z), 6.62 \sim 7.65(\mathrm{~m}, 16 \mathrm{H}, E$ and $Z) ;{ }^{13} \mathrm{C} \mathrm{NMR}\left(50 \mathrm{MHz}, \mathrm{CDCl}_{3}\right) \delta$ $13.7,14.1,55.1,61.5,62.0,113.7,113.9,121.6,127.8,128.7,128.7,128.8$, $129.4,129.9,130.3,131.8,131.8,157.1$.

(Z/E)-Methyl 2-[2,2,2-trifluoro-N-(4-methoxylphenyl)acetimidoyloxy]-3-(4-phenyl)phenyl-2-propenoate (6Zg/6Eg).

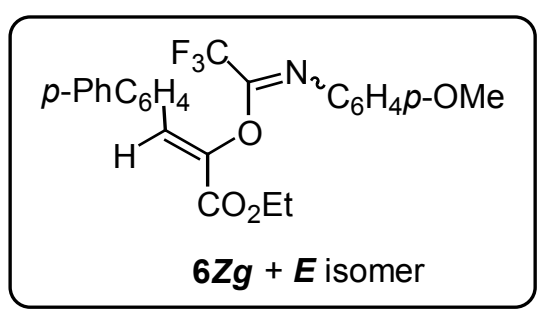

$141.6,142.6,155.0,157.1$.

An inseparable mixture; TLC $R_{f}$ (petroleum ether : acetone $\left.30: 1\right)=$ 0.24; ${ }^{1} \mathrm{H}$ NMR $\left(200 \mathrm{MHz}, \mathrm{CDCl}_{3}\right) \delta 1.18(\mathrm{t}, J=7.2 \mathrm{~Hz}, 1.1 \mathrm{H}, E), 1.33(\mathrm{t}, J$ $=7.2 \mathrm{~Hz}, 1.3 \mathrm{H}, Z), 3.77(\mathrm{~s}, 1.1 \mathrm{H}, E), 3.79(\mathrm{~s}, 0.9 \mathrm{H}, Z), 4.13 \sim 4.32(\mathrm{~m}, 2 \mathrm{H}, E$ and $Z$ ), 6.69 7.74 (m, 14H, $E$ and $Z$ ); $\left.{ }^{13} \mathrm{C} \mathrm{NMR} \mathrm{(50} \mathrm{MHz,} \mathrm{CDCl}_{3}\right) \delta 13.8$, $14.1,55.3,55.4,61.6,62.0,113.7,114.0,121.6,126.6,127.0,127.0,127.4$, $127.6,127.9,128.8,128.9,130.2,130.7,130.8,130.9,138.0,139.9,140.3$, 
(Z/E)-Ethyl 2-[2,2,2-Trifluoro-N-(4-nitrophenyl)acetimidoyloxy]-2-nonenoate (6Zh/6Eh).

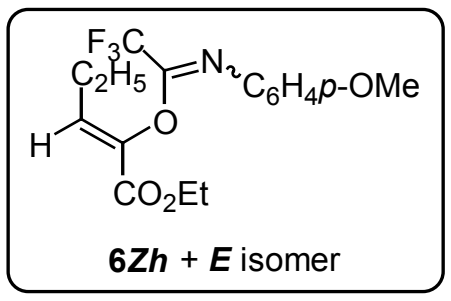

$144.4,149.2,149.4,160.7,160.7$.

An inseparable mixture; TLC $R_{f}$ (petroleum ether : acetone $\left.60: 1\right)=0.36 ;{ }^{1} \mathrm{H}$ NMR (200 MHz, $\left.\mathrm{CDCl}_{3}\right) \delta 1.08(\mathrm{t}, J=7.2 \mathrm{~Hz}, 3 \mathrm{H}, E), 1.35(\mathrm{t}, J=7.2 \mathrm{~Hz}, 3 \mathrm{H}, Z)$, $2.20 \sim 2.36(\mathrm{~m}, 0.9 \mathrm{H}, E), 2.52 \sim 2.67(\mathrm{~m}, 1.2 \mathrm{H}, Z), 4.22 \sim 4.35(\mathrm{~m}, 2 \mathrm{H}, E$ and $Z), 6.14$ $(\mathrm{t}, J=7.8 \mathrm{~Hz}, 0.5 \mathrm{H}, Z), 6.55(\mathrm{t}, J=7.8 \mathrm{~Hz}, 0.3 \mathrm{H}, E), 6.86 \sim 6.93(\mathrm{~m}, 2 \mathrm{H}, E$ and $Z$ ), 8.12 8.20 (m, 2H, $E$ and $Z) ;{ }^{13} \mathrm{C}$ NMR (75 $\left.\mathrm{MHz}, \mathrm{CDCl}_{3}\right) \delta 12.5,13.3,14.0,19.4$, $20.3,61.6,61.8,119.9,120.0,120.4,124.6,125.0,133.5,136.6,138.4,144.3$,

(Z)-Ethyl 2-[2,2,2-Trifluoro-N-(4-nitrophenyl)acetimidoyloxy]-2-nonenoate (6Zi).

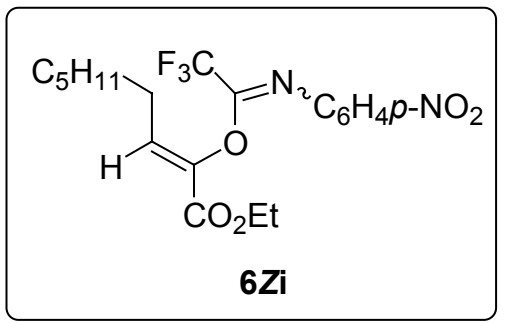

TLC $R_{f}$ (petroleum ether : EtOAc $\left.60: 1\right)=0.41$; IR (film) 2931, 2859, $1728,1657,1593,1519,1375,1330,1211,1164,1137,1110,1022,930,852$, $753695 \mathrm{~cm}^{-1} ;{ }^{1} \mathrm{H}$ NMR $\left(300 \mathrm{MHz}, \mathrm{CDCl}_{3}\right) \delta 0.88(\mathrm{t}, J=6.6 \mathrm{~Hz}, 3 \mathrm{H})$, $1.29 \sim 1.45(\mathrm{~m}, 11 \mathrm{H}), 2.54 \sim 2.62(\mathrm{~m}, 2 \mathrm{H}), 4.30(\mathrm{q}, J=7.2 \mathrm{~Hz}, 2 \mathrm{H}), 6.16(\mathrm{t}, J=$ $7.8 \mathrm{~Hz}, 1 \mathrm{H}), 6.91(\mathrm{~d}, J=8.7 \mathrm{~Hz}, 2 \mathrm{H}), 8.20(\mathrm{~d}, J=8.7 \mathrm{~Hz}, 2 \mathrm{H}){ }^{13} \mathrm{C}$ NMR $(75$ $\left.\mathrm{MHz}, \mathrm{CDCl}_{3}\right) \delta 14.0,14.1,22.5,26.8,28.8,28.9,31.5,61.5,115.9\left(\mathrm{q}, J_{C-F}=\right.$ $\left.283.1 \mathrm{~Hz}, \mathrm{CF}_{3}\right), 120.0,124.6,135.5,138.1,144.4,149.4,160.8$; EI-MS $(\mathrm{m} / \mathrm{z}$, relative intensity): $416\left(\mathrm{M}^{+}, 4\right), 343$ (17), 331 (42), 299 (16), 217 (100), 171 (20), 122 (33), 92 (16); Anal. calcd for $\mathrm{C}_{19} \mathrm{H}_{23} \mathrm{~F}_{3} \mathrm{~N}_{2} \mathrm{O}_{5}$ : C, 54.80; H, 5.57; N, 6.73. Found: C, 55.00; H, 5.73; N, 6.46.

(Z/E)-Methyl 2-[2,2,2-Trifluoro-N-(4-nitrophenyl)acetimidoyloxy]-3-phenyl-2-propenoate (6Zj/6Ej)

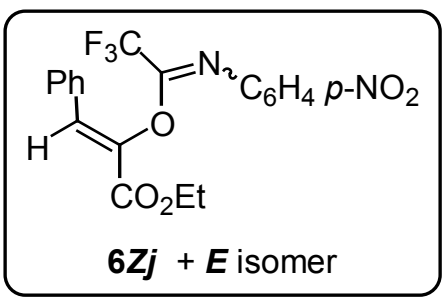

$148.9,149.0,160.6,161.2$.

An inseparable mixture; TLC $R_{f}$ (petroleum ether : EtOAc $\left.30: 1\right)=0.22 ;{ }^{1} \mathrm{H}$ NMR $\left(300 \mathrm{MHz}, \mathrm{CDCl}_{3}\right) \delta 1.70(\mathrm{t}, J=7.2 \mathrm{~Hz}, 1.0 \mathrm{H}, E), 1.37(\mathrm{t}, J=7.2 \mathrm{~Hz}, 3 \mathrm{H}$, $Z$ ), $4.21(\mathrm{q}, J=7.2 \mathrm{~Hz}, 0.7 \mathrm{H}, E), 4.33$ (q, $J=7.2 \mathrm{~Hz}, 2 \mathrm{H}, Z), 6.80 \sim 8.21(\mathrm{~m}, 14 \mathrm{H}$, $E$ and $Z) ;{ }^{13} \mathrm{C}$ NMR $\left(75 \mathrm{MHz}, \mathrm{CDCl}_{3}\right) \delta 13.6,14.0,61.7,62.2,115.9\left(\mathrm{q}, J_{C-F}=\right.$ $\left.282.0 \mathrm{~Hz}, \mathrm{CF}_{3}\right), 119.8,119.9,124.4,124.6,126.7,127.2,127.7,128.0,128.3$, $129.0,129.2$, 129.5, 130.2, 130.5, 130.6, 131.2, 131.3, 137.2, 138.1, 144.5, 148.7,

(Z)-Ethyl 2-[2,2,2-Trifluoro-N-(3,4-dichlorophenyl)acetimidoyloxy]-2-pentenoate (6Zk).

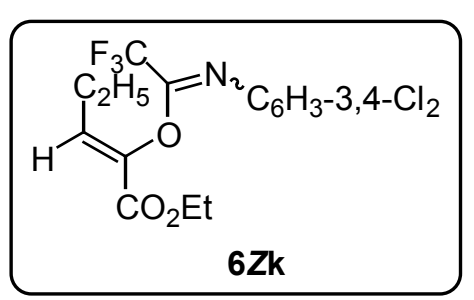

TLC $R_{f}$ (petroleum ether : EtOAc $\left.30: 1\right)=0.56$; IR (film) 2982, 1724, 1656, 1593, 1556, 1466, 1375, 1334, 1227, 1209, 1140, 1124, 1030, 954, 814, 743, 698 $\mathrm{cm}^{-1} ;{ }^{1} \mathrm{H}$ NMR $\left(300 \mathrm{MHz}, \mathrm{CDCl}_{3}\right) \delta 1.06(\mathrm{~s}, 3 \mathrm{H}), 1.33(\mathrm{t}, J=7.2 \mathrm{~Hz}, 3 \mathrm{H}), 2.57$ $(\mathrm{s}, 2 \mathrm{H}), 4.27(\mathrm{q}, J=7.2 \mathrm{~Hz}, 2 \mathrm{H}), 6.07(\mathrm{t}, J=7.8 \mathrm{~Hz}, 1 \mathrm{H}), 6.56 \sim 6.80(\mathrm{~m}, 1 \mathrm{H})$, $6.94(\mathrm{~s}, 1 \mathrm{H}), 7.33 \sim 7.36(\mathrm{~m}, 1 \mathrm{H}) ;{ }^{13} \mathrm{C} \mathrm{NMR}\left(75 \mathrm{MHz}, \mathrm{CDCl}_{3}\right) \delta 13.4,14.0,20.4$, $61.5,119.4,121.8,128.2,130.3,132.5,136.2,138.0,142.8,160.6$; EI-MS $(m / z$, relative intensity): $383\left(\mathrm{M}^{+}, 6\right), 354$ (27), 240 (100), 145 (45), 109 (11); Anal. calcd for $\mathrm{C}_{15} \mathrm{H}_{14} \mathrm{Cl}_{2} \mathrm{~F}_{3} \mathrm{NO}_{3}$ : C, 46.90; $\mathrm{H}$, 3.67; N, 3.65. Found: C, 47.19; H, 3.78; N, 3.67. 
(Z/E)-Methyl 2-[2,2,2-Trifluoro-N-(3,4-dicholophenyl)acetimidoyloxy]-3-phenyl-2-propenoate (6Zl/6El)

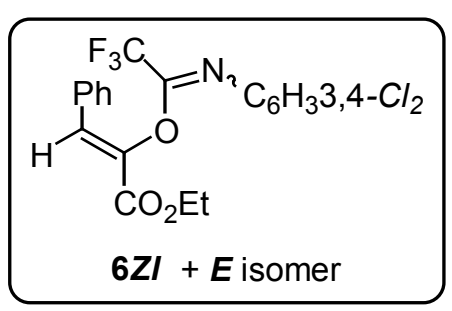

TLC $R_{f}$ (petroleum ether : EtOAc $\left.30: 1\right)=0.38 ;{ }^{1} \mathrm{H}$ NMR $\left(300 \mathrm{MHz}, \mathrm{CDCl}_{3}\right)$ $\delta 1.50(\mathrm{t}, J=7.2 \mathrm{~Hz}, 2.0 \mathrm{H}, E), 1.36(\mathrm{t}, J=7.2 \mathrm{~Hz}, 3 \mathrm{H}, Z), 4.17$ (q, $J=7.2 \mathrm{~Hz}$, $1.3 \mathrm{H}, E), 4.31$ (q, $J=7.2 \mathrm{~Hz}, 2 \mathrm{H}, Z), 6.55 \sim 7.62$ (m, $15 \mathrm{H}, E$ and $Z$ ); ${ }^{13} \mathrm{C}$ NMR (75 $\left.\mathrm{MHz}, \mathrm{CDCl}_{3}\right) \delta 13.6,14.1,61.7,62.2,119.3,121.6,126.7,127.9,128.4,129.0$, $129.1,129.8,130.1,130.3,130.4,131.4,131.5,132.5,132.7,137.5,142.2,161.3$.

(Z)-Ethyl 2-(2,2,2-trichloro-N-methylacetimidoyloxy)-2-pentenoate (11Zb).

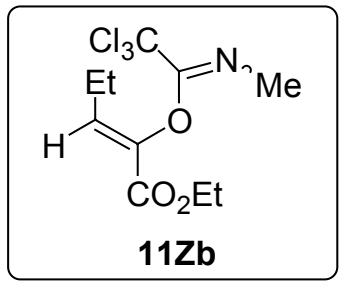

TLC $R_{f}$ (petroleum ether : acetone $15: 1$ ) = 0.44; IR (film) 2976, 1728, 1693, 1643, 1463, 1374, 1312, 1231, 1144, 1105, 1062, 1035, 936, 877, 826, 793, $742 \mathrm{~cm}^{-1} ;{ }^{1} \mathrm{H}$ NMR $\left(200 \mathrm{MHz}, \mathrm{CDCl}_{3}\right) \delta 1.08(\mathrm{t}, J=7.2 \mathrm{~Hz}, 3 \mathrm{H}), 1.33(\mathrm{t}, J=7.2 \mathrm{~Hz}, 3 \mathrm{H}), 2.51 \sim 2.66(\mathrm{~m}, 2 \mathrm{H})$, 3.25 (s, 3H), 4.29 (q, $J=7.2 \mathrm{~Hz}, 2 \mathrm{H}), 5.81(\mathrm{t}, J=7.5 \mathrm{~Hz}, 1 \mathrm{H}) ;{ }^{13} \mathrm{C}$ NMR $(50 \mathrm{MHz}$, $\left.\mathrm{CDCl}_{3}\right) \delta 13.8,14.0,20.1,36.2,61.3,128.3,140.3,148.9,161.6$; EI-MS $(\mathrm{m} / z$, relative intensity): $302\left[(\mathrm{M}+1)^{+}, 4.98\right], 272$ (8), 256 (3), 158 (100), 117 (59), 99 (4), 82 (18), 55

(27), 39 (24), 29 (91); Anal. calcd for $\mathrm{C}_{10} \mathrm{H}_{14} \mathrm{Cl}_{3} \mathrm{NO}_{3}$ : C, 39.69; H, 4.66; N, 4.63;. Found: C, 39.85; H, 4.87; N, 4.50.

Figure 1. X-ray structure of $\mathbf{3 a}$ and $\mathbf{3 b}$

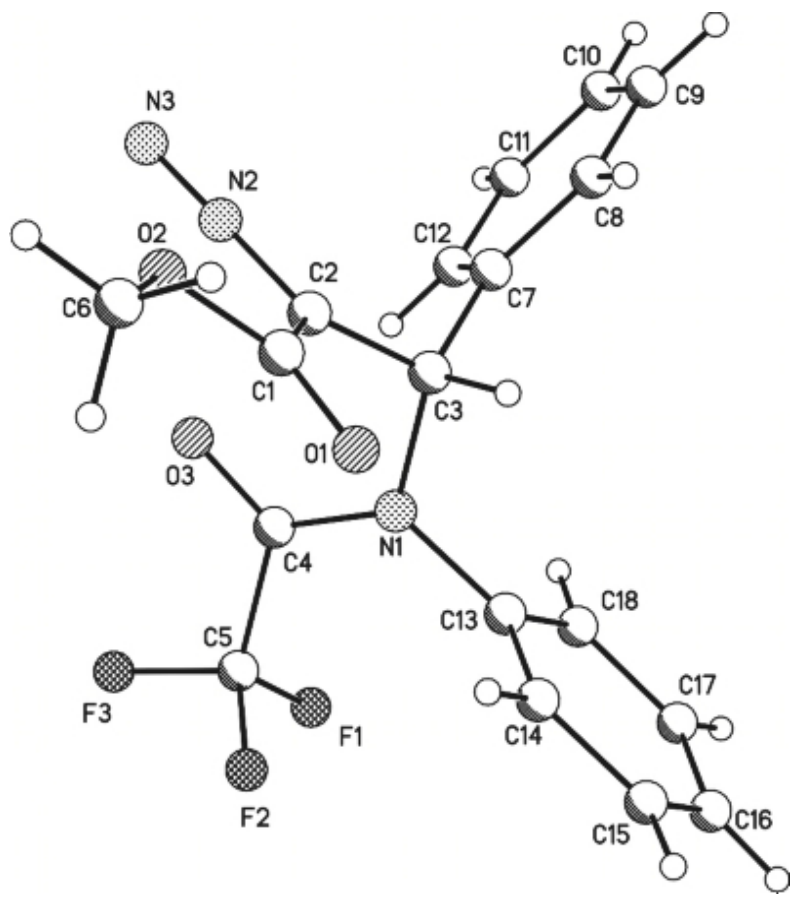




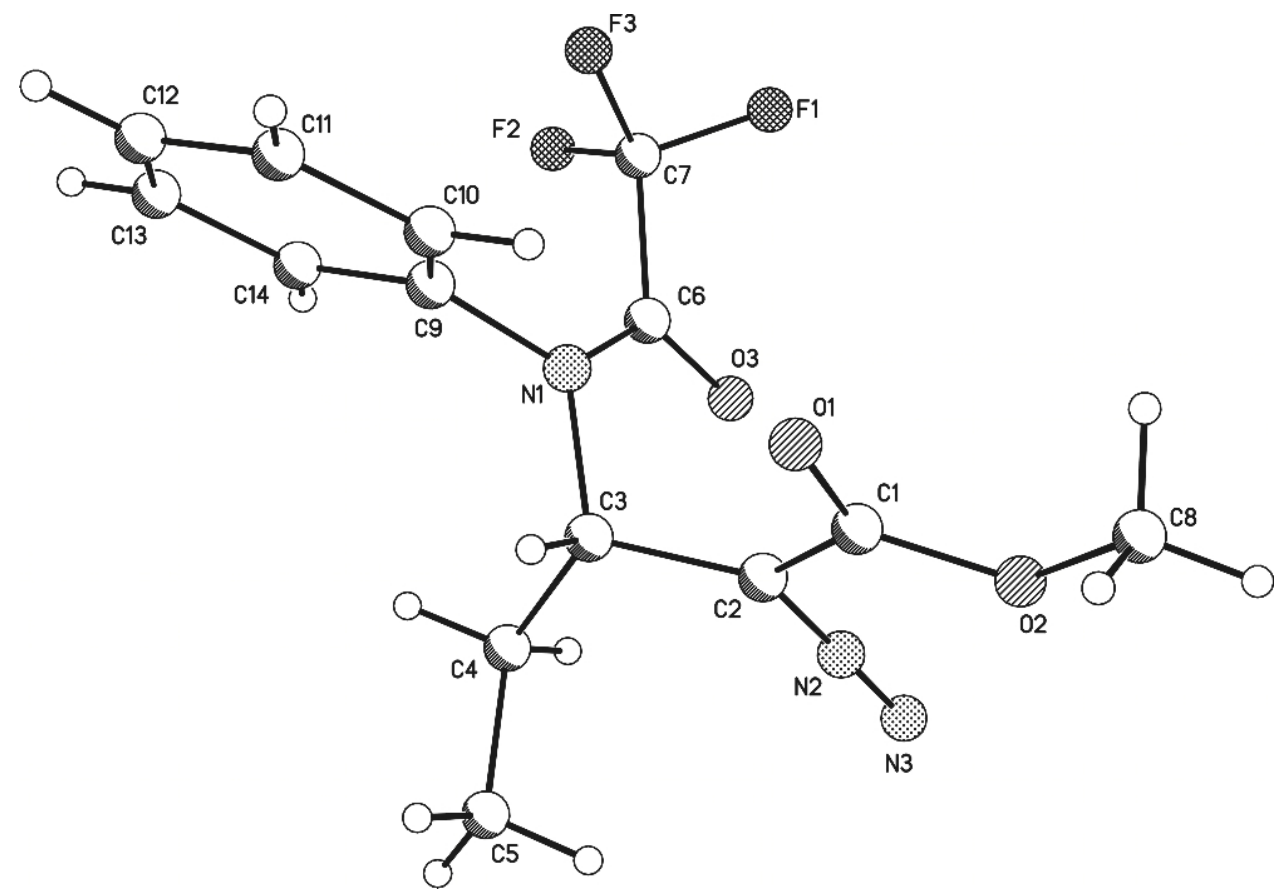

3b

Figure 2. X-ray structure of $6 E \mathrm{a}$ and $6 Z \mathrm{a}$

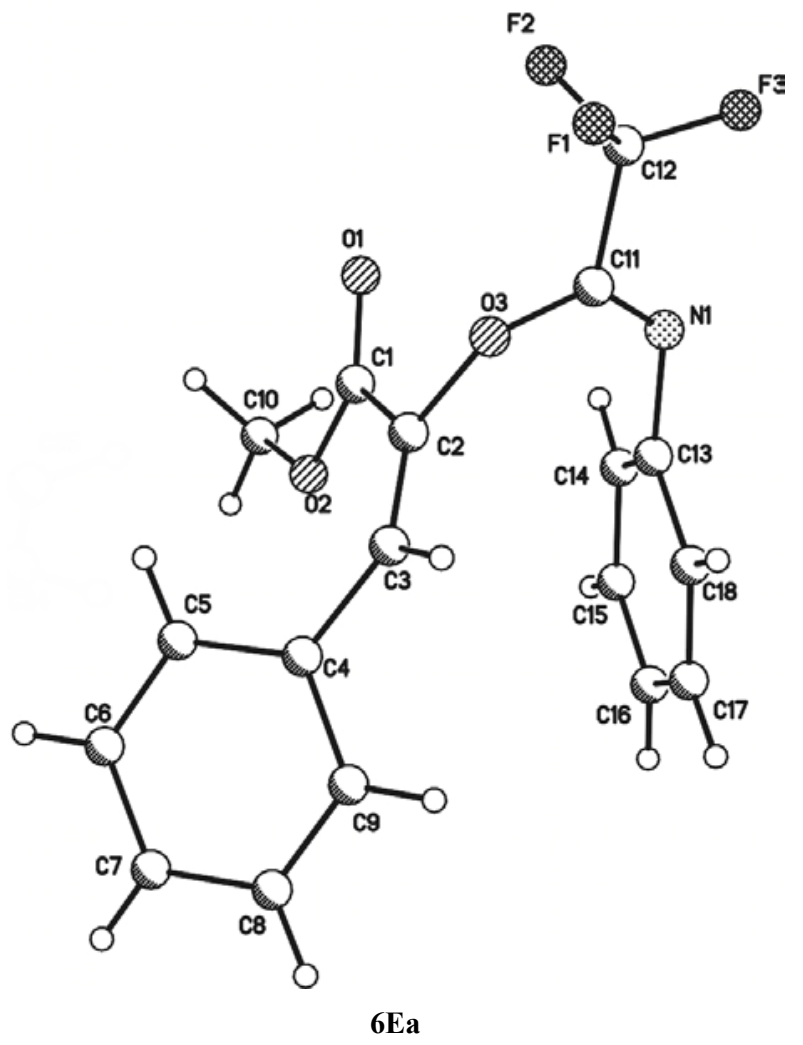




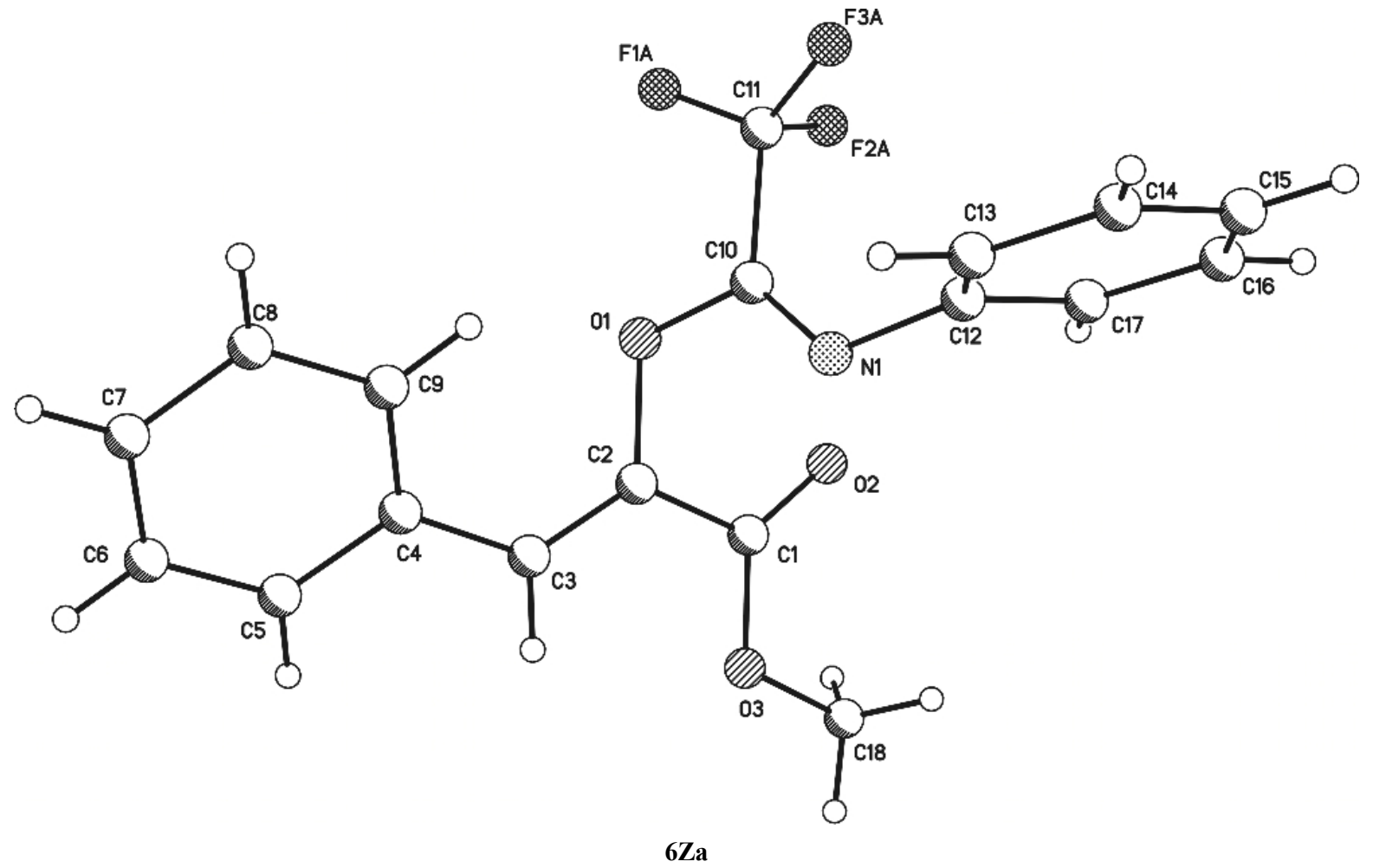

